process that can produce patterns and functional devices that establish new resolution benchmarks for liquid printing, significantly exceeding those of established ink-jet technologies," said John Rogers, a Founder Professor of Materials Science and Engineering, and corresponding author of an article published in the October 1 issue of Nature Materials (p. 782; DOI: 10.1038/nmat974).

This type of e-jet printing could be used for large-area circuits, displays, photovoltaic modules and related devices, and other application possibilities in security, biotechnology, and photonics, Rogers said.

"As an industrial process, this work opens up the possibility for low-cost and high-performance printed electronics and other systems that involve materials that cannot be manipulated with more common patterning methods derived from microelectronics fabrication," said Placid Ferreira, the Grayce Wicall Gauthier Professor of Mechanical Science and Engineering, the director of the center and a key member of the team.

Unlike conventional ink-jet printers, which use heat or mechanical vibrations to launch liquid droplets through a nozzle, e-jet printing uses electric fields to pull the fluid out. Although the concept of electric-field induced flow is not new, the way the research team has exploited this phenomenon with nanoscale nozzles and precision control of electric fields to achieve unprecedented levels of resolution is an important advance.

The researchers' e-jet printing head consists of a gold-coated microcapillary nozzle (with a diameter as small as $300 \mathrm{~nm}$ ) mounted on a computer-controlled mechanical support. An organic, Teflon-like coating on the gold ensures the ink flows cleanly out the nozzle toward the target. Tiny droplets of ink eject onto a moving substrate to produce printed patterns. Lines with widths as narrow as $700 \mathrm{~nm}$, and dots as small as $250 \mathrm{~nm}$, can be achieved in this fashion.

As a demonstration of electronic device fabrication by e-jet printing, thin-film transistors that use aligned arrays of singlewalled carbon nanotubes as the semiconductor and e-jet-printed source and drain electrodes were printed on flexible plastic substrates. The transistors were fully operational, with properties comparable to similar devices fabricated with conventional photolithographic methods.

The team also demonstrated that e-jet printing could be extended to a variety of functional organic and inorganic inks, including suspensions of solid objects (such as nanoscale silicon rods) with res- olutions again extending to the submicron range.

Because the nozzles are routed directly to ink reservoirs, e-jet printing has the capability to deliver large ink volumes to a surface and offers the ability to perform preprocessing on the inks before printing, Rogers said.

\section{Efficient Electronic Devices Obtained through Molecular-Level "Contamination"}

One way to improve something is to "contaminate" it slightly. For example, the modern electronics industry is based on the practice of taking a relatively "pure" semiconductor, such as silicon, and purposely introducing small amounts of "impurities" into it. It is these impurities that enable the conductance of electricity through the semiconductor and, thus, allow control over the electronic properties of the material. Recently, a group of scientists at the Weizmann Institute of Science led by D. Cahen, together with C. Chan of Princeton University and their colleagues, implemented this technique in the field of molecular electronics. Their work was reported in the June 20 issue of the Journal of the American Chemical Society (p. 7494; DOI: 10.1021/ja071960p).

Molecular electronics is based on single molecules or on layers that are no more than one molecule thick. The problem with using these monolayers for electronic purposes is that they form a delicate system that is hard to manipulate precisely, and until recently, it was unclear whether it was even possible to dope them.

The current research demonstrates, however, that such "contamination" is indeed possible.

The first stage involves cleaning the molecular layer of its defects through long processes of drying, cleaning, and removing oxygen. In this case, the researchers used simple organic molecules, that is, $\mathrm{CH}_{2}-\left(\mathrm{CH}_{2}\right)_{12}-\mathrm{CH}_{3}$ hydrocarbon chains (similar to octane found in petrol), which are electrically insulating. Electrical transport measurements through $\mathrm{Si}-\mathrm{CH}_{2}-\left(\mathrm{CH}_{2}\right)_{12}-\mathrm{CH}_{3} / / \mathrm{Hg}$ junctions demonstrated that the layers acted as ideal insulators. Characterization of the resulting "clean" surface showed that, although the resulting molecular system included unavoidable impurities to a certain degree, those impurities did not dictate the system's electrical behavior.

To dope the clean surface, the scientists irradiated it with UV light or weak electron beams. As a result, chemical changes occurred within the system: double (as opposed to single) chemical bonds were created between the carbon atoms that assemble into the molecular chains that make up the molecular layer. It is these bonds that ultimately influence electronic transport through the molecules (see figure).

Cahen said, "The method that we have developed allows us to start working with a system that is made up of a uniform layer of 'pure' molecules, which dominates the structure's overall electrical properties.

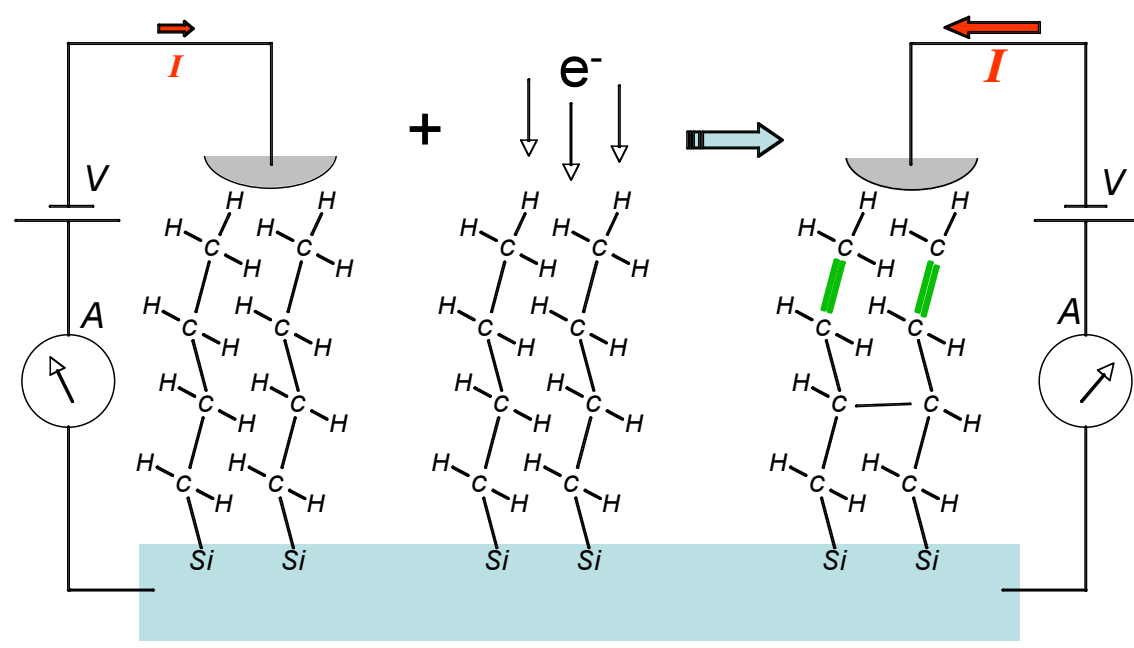

Figure 1. A single layer of chains of hydrocarbons (molecules with hydrogen, $\mathrm{H}$, bound to carbon, C) on a surface of silicon (light blue). Exposing the molecules to a weak beam of electrons (center) causes chemical changes (loss of some hydrogen atoms), creating double bonds between carbon atoms (right, green). As a result, the material's electronic transport properties change, changing the mechanism of transport and increasing the electric current by a factor of $10-100$. 
After establishing such an ideal system, it is then possible to 'dope' the system by introducing impurities into it, thereby allowing control over its electronic transport properties according to one's specific needs."

\section{DNA Used as a Template for Nanolithography}

DNA is one of the most popular building blocks of nanotechnology and is commonly used to construct ordered nanoscale structures with controlled architectures. For the most part, DNA is looked upon as a promising building block for fabricating microelectronic circuits from the bottom up. Now, A. Woolley and H. Becerril of Brigham Young University are proposing the marriage of DNA self-assembly with standard microfabrication and lithography tools to form features such as nanochannels, nanowires, and nanoscale trenches. This discovery, reported in the September 3 issue of Small (p. 1534; DOI: 10.1002/smll.
200700240) could open new avenues for nanofabrication at dimensions not accessible by conventional optical lithography.

The researchers have developed a method to use DNA molecules as templates to define patterns on substrates. They deposited metal films over DNA molecules aligned on a substrate. The DNA molecules essentially act as nanostencils to define sub-10-nm-sized patterns on the substrate. The researchers call this process "DNA shadow nanolithography" because the metal film is deposited at an angle and the shadow cast by the DNA molecules defines the dimensions of the features on the substrate.

Anisotropic etching of the patterned surfaces using reactive gas plasmas, a commonly used fabrication tool in the semiconductor industry, yields highaspect-ratio trenches on the substrate. The trenches can be sealed at the top to form continuous enclosed nanochannels.
Alternatively, the trenches can be chemically functionalized and used as templates for the deposition of metal nanowires such as those of nickel, copper, or silver. The templated trenches and nanowires have lateral dimensions of less than $30 \mathrm{~nm}$ and can be tailored to be less than $10 \mathrm{~nm}$. The exact dimensions of the trenches can be varied by tuning the angle of deposition and the thickness of the deposited film.

The researchers said that it should be possible to transfer complex patterns onto substrates using surface-aligned DNA molecules. "One remarkable aspect of this technology is that it utilizes the patterning ability of DNA without requiring the nucleic acid to remain in the final construct," said Woolley, adding that the nanostructures fabricated by DNA shadow nanolithography could find use as nanofluidic channels and chemical sensors.
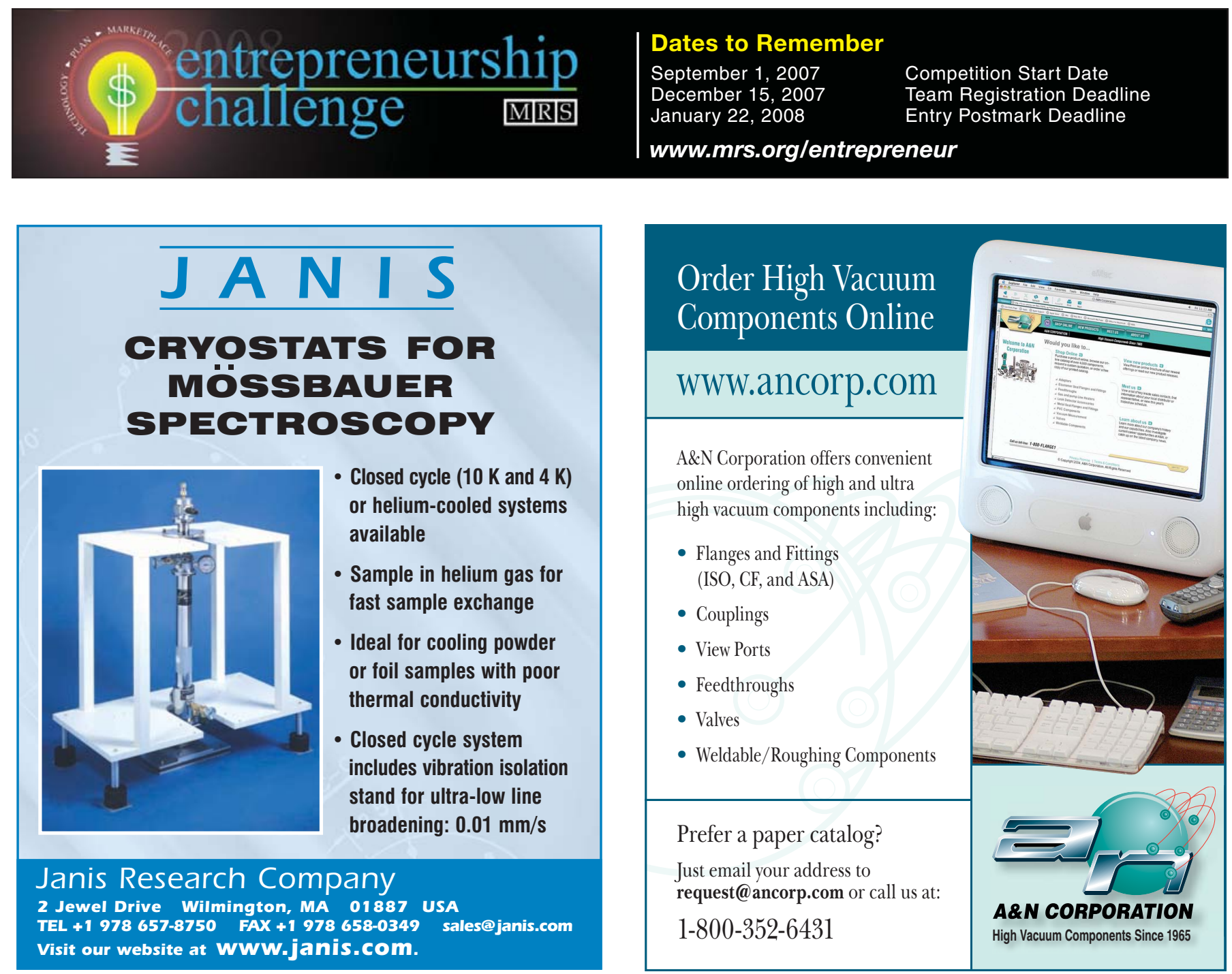

Order High Vacuum
Components Online
WWW.anCOrP.COM
online ordering of high and ultra
high vacuum components including:
- Flanges and Fittings
- Couplings
- View Ports
- Feedthroughs
- Valves
- Weldable/Roughing Components
Pust email your address to
1-800-35est@ancorp.com or call us at:

